\title{
A NEW LICHEN RECORD FOR TURKEY AND ADDITIONS TO THE LICHEN DIVERSITY OF THE GIRESUN PROVINCE (TURKEY)
}

\author{
K. KINALIOĞLU ${ }^{1}$ and A. Aptroot ${ }^{2}$ \\ ${ }^{1}$ Department of Biology, Faculty of Science and Arts, Giresun University, Güre Yerleşkesi, \\ Giresun, Turkey; E-mail: kkinalioglu@hotmail.com \\ ${ }^{2}$ Adviesbureau voor Bryologie en Lichenologie, G.v.d. Veenstraat 107, NL-3762 XK Soest, \\ The Netherlands; E-mail: andreaptroot@gmail.com
}

(Received 4 May, 2017; Accepted 30 October, 2017)

\begin{abstract}
A list of 136 lichen species from the Giresun province (Turkey) is reported. Among them, 73 are new records for province, and Acarospora molybdina is new to Turkey. Here with, the infraspecific taxa for the province rise from 475 to 548 . Locality and substrate data is presented for each taxa. Brief taxonomic description and comments are also provided for the Acarospora molybdina.
\end{abstract}

Key words: Ascomycota, biodiversity, Giresun province, lichens, Turkey

\section{INTRODUCTION}

The first lichen record from Giresun province, which lies in East Black Sea Region of Turkey, was published by Steiner (1909) based on materials collected by Handel-Mazzetti. In last three decades, lichen diversity of the province was attracted the interest of lichenologists, and with the rise of the lichenology in Turkey, many floristic records were published from the area (Aslan and Yazıcı 2006, Aslan et al. 2002, Çobanoğlu 2011, Duman and Yurdakulol 2007, Halıcı and Şenkardeşler 2009, John 2007, John and Breuss 2004, Kinalıoğlu 2005, 2006, 2008, 2009, 2010a, b, c, Kınalıoğlu and Aptroot 2010, 2011, Kınalığlu and Engin 2004, Kınalığlu and Uzun 2016, Küçük 1990, Sezer 2016, Yazıc1 and Aptroot 2008, Yazıc1 and Aslan 2005, 2006).

The aim of this study is to contribute to the lichen flora of the province. Although Giresun is one of the most examined cities of Turkey in point of lichen diversity, our study shows clearly that the city still needs to be observed.

\section{MATERIALS AND METHODS}

Specimens were collected from 52 localities (Fig. 1) visited between 2004 and 2015. All specimens have been identified using routine microscope techniques and traditional chemical reagents with the aid of various lichen litera- 
tures (e.g. Brodo et al. 2001, Dobson 2011, Smith et al. 2009, Wasser and Nevo 2005, Wirth 1995, Wirth et al. 2013). The list of sampling localities are presented below. Samples are placed in the herbarium of the Biology Department, Faculty of Science and Arts, Giresun University, Giresun, Turkey (GUB).

\section{Sampling localities}

1. Bulancak, N of Bayındır village, $1112 \mathrm{~m}, 40^{\circ} 43^{\prime} 22^{\prime \prime} \mathrm{N}, 38^{\circ} 08^{\prime} 12^{\prime \prime} \mathrm{E}, 05.05 .2006$.

2. Centre, Ayvasil place, sea shore, $7 \mathrm{~m}, 40^{\circ} 55^{\prime} 35^{\prime \prime} \mathrm{N}, 38^{\circ} 18^{\prime} 44^{\prime \prime} \mathrm{E}, 25.05 .2006$.

3. Centre, SE slope of Gedikkaya hill, $190 \mathrm{~m}, 40^{\circ} 54^{\prime} 35^{\prime \prime} \mathrm{N}, 38^{\circ} 24^{\prime} 48^{\prime \prime} \mathrm{E}, 10.06 .2006$.

4. Centre, SW of Giresun city centre, SE of Boztekke village, $21 \mathrm{~m}, 40^{\circ} 54^{\prime} 34^{\prime \prime} \mathrm{N}, 38^{\circ} 19^{\prime} 35^{\prime \prime}$ E, 11.08.2008.

5. Centre, SW of city centre, Boztekke village, $43 \mathrm{~m}, 40^{\circ} 54^{\prime} 10^{\prime \prime} \mathrm{N}, 38^{\circ} 19^{\prime} 16^{\prime \prime} \mathrm{E}, 10.04 .2010$.

6. Centre, Aksu district, near the stream, $19 \mathrm{~m}, 40^{\circ} 53^{\prime} 30^{\prime \prime} \mathrm{N}, 38^{\circ} 26^{\prime} 26^{\prime \prime} \mathrm{E}, 16.05 .2015$.

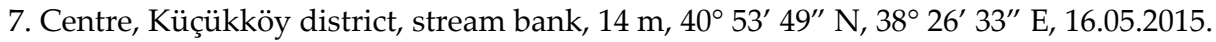

8. Centre, Çaykara district, stream bank, $26 \mathrm{~m}, 40^{\circ} 53^{\prime} 14^{\prime \prime} \mathrm{N}, 38^{\circ} 26^{\prime} 23^{\prime \prime} \mathrm{E}, 16.05 .2015$.

9. Centre, Teyyaredüzü district, $13 \mathrm{~m}, 40^{\circ} 54^{\prime} 42^{\prime \prime} \mathrm{N}, 38^{\circ} 20^{\prime} 12^{\prime \prime} \mathrm{E}, 04.05 .2015$.

10. Dereli, Tamdere village, $1690 \mathrm{~m}, 40^{\circ} 30^{\prime} 14^{\prime \prime} \mathrm{N}, 38^{\circ} 21^{\prime} 02^{\prime \prime} \mathrm{E}, 06.05 .2005$.

11. Dereli, Tepeköknarlı village, $605 \mathrm{~m}, 40^{\circ} 47^{\prime} 28^{\prime \prime} \mathrm{N}, 38^{\circ} 26^{\prime} 44^{\prime \prime}$ E, 14.04.2005.

12. Dereli, northern slopes of Karagöl mountains, 2810 m, $40^{\circ} 31^{\prime} 03^{\prime \prime} \mathrm{N}, 38^{\circ} 10^{\prime} 02^{\prime \prime}$ E, 29.07.2007.

13. Dereli, Karagöl Mountains, 3050 m, 40 $41^{\prime} 24^{\prime \prime}$ N, 38 09' 10" E, 29.07.2007.

14. Dereli, Aymaç place, $1827 \mathrm{~m}, 40^{\circ} 34^{\prime} 18^{\prime \prime} \mathrm{N}, 38^{\circ} 24^{\prime}$ 50" E, 29.05.2008.

15. Dereli, E of Kuzu gölü yaylası, $2143 \mathrm{~m}, 40^{\circ} 34^{\prime} 42^{\prime \prime}$ N, $38^{\circ} 29^{\prime} 21^{\prime \prime}$ E, 09.08.2008.

16. Dereli, N of Kümbet yaylass, 1650 m, $40^{\circ} 33^{\prime} 09^{\prime \prime}$ N, $38^{\circ} 27^{\prime} 36^{\prime \prime}$ E, 10.08.2008.

17. Dereli, SE of Yüce village, $1345 \mathrm{~m}, 40^{\circ} 37^{\prime} 37^{\prime \prime} \mathrm{N}, 38^{\circ} 28^{\prime} 11^{\prime \prime} \mathrm{E}, 17.05 .2008$.

18. Espiye, Gülburnu, sea shore, $1 \mathrm{~m}, 40^{\circ} 57^{\prime} 50^{\prime \prime} \mathrm{N}, 38^{\circ} 39^{\prime} 14^{\prime \prime} \mathrm{E}, 10.06 .2006$.

19. Espiye, $\mathrm{S}$ of Arpacik village, $196 \mathrm{~m}, 40^{\circ} 53^{\prime} 01^{\prime \prime} \mathrm{N}, 38^{\circ} 46^{\prime} 33^{\prime \prime} \mathrm{E}, 08.11 .2014$.

20. Espiye, SW of Bahçecik village, $220 \mathrm{~m}, 40^{\circ} 53^{\prime} 40^{\prime \prime} \mathrm{N}, 38^{\circ} 44^{\prime} 17^{\prime \prime} \mathrm{E}, 08.11 .2014$.

21. Espiye, Çepniköy village, $465 \mathrm{~m}, 40^{\circ} 51^{\prime} 25^{\prime \prime} \mathrm{N}, 38^{\circ} 43^{\prime} 59^{\prime \prime} \mathrm{E}, 08.11 .2014$.

22. Espiye, $\mathrm{S}$ of Direkbükü village, $167 \mathrm{~m}, 40^{\circ} 51^{\prime} 10^{\prime \prime} \mathrm{N}, 38^{\circ} 46^{\prime} 12^{\prime \prime} \mathrm{E}, 08.11 .2014$.

23. Espiye, E of Ericek village, $955 \mathrm{~m}, 40^{\circ} 42^{\prime} 58^{\prime \prime} \mathrm{N}, 38^{\circ} 43^{\prime} 00^{\prime \prime} \mathrm{E}, 08.11 .2014$.

24. Espiye, E of Güneyköy village, $560 \mathrm{~m}, 40^{\circ} 52^{\prime} 19^{\prime \prime} \mathrm{N}, 38^{\circ} 44^{\prime} 45^{\prime \prime} \mathrm{E}, 08.11 .2014$.

25. Espiye, Kizlldere district, 40 m, 40 $54^{\prime} 36^{\prime \prime}$ N, $38^{\circ} 44^{\prime} 55^{\prime \prime}$ E, 08.11.2014.

26. Espiye, Soğukpınar town, $624 \mathrm{~m}, 40^{\circ} 49^{\prime} 08^{\prime \prime} \mathrm{N}, 38^{\circ} 43^{\prime} 56^{\prime \prime} \mathrm{E}, 08.11 .2014$.

27. Espiye, Kurugeris village, $635 \mathrm{~m}, 40^{\circ} 49^{\prime} 30^{\prime \prime} \mathrm{N}, 38^{\circ} 46^{\prime} 59^{\prime \prime} \mathrm{E}, 08.11 .2014$.

28. Espiye, $\mathrm{S}$ of Yeşilköy village, $285 \mathrm{~m}, 40^{\circ} 50^{\prime} 01^{\prime \prime} \mathrm{N}, 38^{\circ} 45^{\prime} 59^{\prime \prime} \mathrm{E}, 08.11 .2014$.

29. Espiye, Yeşilyurt village, $472 \mathrm{~m}, 40^{\circ} 53^{\prime} 05^{\prime \prime} \mathrm{N}, 38^{\circ} 42^{\prime} 51^{\prime \prime} \mathrm{E}, 08.11 .2014$.

30. Espiye, E of Gülburnu, sea shore, $1 \mathrm{~m}, 40^{\circ} 57^{\prime} 04^{\prime \prime} \mathrm{N}, 38^{\circ} 40^{\prime} 20^{\prime \prime} \mathrm{E}, 17.05 .2015$.

31. Keşap, between Geçit village and Erköy village, $950 \mathrm{~m}, 40^{\circ} 44^{\prime} 04^{\prime \prime} \mathrm{N}, 38^{\circ} 35^{\prime} 16^{\prime \prime}$ E, 25.08. 2004.

32. Keşap, SE of Ceylanpınar village, stream bank, $604 \mathrm{~m}, 40^{\circ} 47^{\prime} 34^{\prime \prime} \mathrm{N}, 38^{\circ} 32^{\prime} 36^{\prime \prime} \mathrm{E}$, 25.08.2004.

33. Keşap, Dokuztepe village, $360 \mathrm{~m}, 40^{\circ} 52^{\prime} 46^{\prime \prime} \mathrm{N}, 38^{\circ} 31^{\prime} 30^{\prime \prime} \mathrm{E}, 03.11 .2005$.

34. Keşap, Değirmenağzı village, $12 \mathrm{~m}, 40^{\circ} 58^{\prime} 24^{\prime \prime} \mathrm{N}, 38^{\circ} 38^{\prime} 34^{\prime \prime} \mathrm{E}, 12.02 .2006$.

35. Keşap district, Değirmenağzı village, sea shore, $1 \mathrm{~m}, 40^{\circ} 58^{\prime} 20^{\prime \prime} \mathrm{N}, 38^{\circ} 37^{\prime} 23^{\prime \prime} \mathrm{E}$, 11.04.2010. 
36. Keşap district, Değirmenağzı village, sea shore, $4 \mathrm{~m}, 40^{\circ} 58^{\prime} 21^{\prime \prime} \mathrm{N}, 38^{\circ} 37^{\prime} 22^{\prime \prime} \mathrm{E}$, 11.04.2010.

37. Keşap, Değirmenağzı village, sea shore, $2 \mathrm{~m}, 40^{\circ} 58^{\prime} 23^{\prime \prime} \mathrm{N}, 38^{\circ} 37^{\prime} 30^{\prime \prime} \mathrm{E}, 19.05 .2015$.

38. Piraziz, S of Hasanşeyh village, $425 \mathrm{~m}, 40^{\circ} 53^{\prime} 33^{\prime \prime} \mathrm{N}, 38^{\circ} 07^{\prime} 43^{\prime \prime} \mathrm{E}, 30.03 .2006$.

39. Piraziz, E of Gökçeali village, $422 \mathrm{~m}, 40^{\circ} 54^{\prime} 24^{\prime \prime} \mathrm{N}, 38^{\circ} 05^{\prime} 39^{\prime \prime} \mathrm{E}, 30.03 .2006$.

40. Piraziz, W of Çayırköy village, $745 \mathrm{~m}, 40^{\circ} 52^{\prime} 24^{\prime \prime} \mathrm{N}, 38^{\circ} 03^{\prime} 57^{\prime \prime} \mathrm{E}, 30.03 .2006$.

41. Şebinkarahisar, $\mathrm{N}$ of Asarcik village, $1850 \mathrm{~m}, 40^{\circ} 25^{\prime} 10^{\prime \prime} \mathrm{N}, 38^{\circ} 23^{\prime} 33^{\prime \prime} \mathrm{E}, 01.09 .2015$.

42. Şebinkarahisar, S of Eğribel pass, $2111 \mathrm{~m}, 40^{\circ} 26^{\prime} 27^{\prime \prime} \mathrm{N}, 38^{\circ} 23^{\prime} 35^{\prime \prime} \mathrm{E}, 01.09 .2015$.

43. Şebinkarahisar, E of Eğribel pass, $2264 \mathrm{~m}, 40^{\circ} 27^{\prime} 21^{\prime \prime} \mathrm{N}, 38^{\circ} 24^{\prime} 02^{\prime \prime} \mathrm{E}, 01.09 .2015$.

44. Şebinkarahisar, S of Eğribel pass, $2109 \mathrm{~m}, 40^{\circ} 26^{\prime} 28^{\prime \prime} \mathrm{N}, 38^{\circ} 23^{\prime} 34^{\prime \prime} \mathrm{E}, 01.09 .2015$.

45. Şebinkarahisar, between Asarcık village and Şaplıca village, stream bank, $1505 \mathrm{~m}, 40^{\circ}$ $24^{\prime} 13^{\prime \prime} \mathrm{N}, 38^{\circ} 25^{\prime} 09^{\prime \prime} \mathrm{E}, 01.09 .2015$.

46. Şebinkarahisar, W of Turna plain, $2561 \mathrm{~m}, 40^{\circ} 28^{\prime} 32^{\prime \prime} \mathrm{N}, 38^{\circ} 25^{\prime} 20^{\prime \prime} \mathrm{E}, 01.09 .2015$.

47. Şebinkarahisar, NE of Tamzara village, $1243 \mathrm{~m}, 40^{\circ} 19^{\prime} 53^{\prime \prime} \mathrm{N}, 38^{\circ} 26^{\prime} 31^{\prime \prime} \mathrm{E}$, 01.09.2015.

48. Şebinkarahisar, between Baltaşı village and Duman village, $1174 \mathrm{~m}, 40^{\circ} 19^{\prime} 53^{\prime \prime} \mathrm{N}, 38^{\circ}$ 26' 31" E, 01.09.2015.

49. Şebinkarahisar, NE of Duman village, near the Kılıçkaya dam, $1037 \mathrm{~m}, 40^{\circ} 14^{\prime} 18^{\prime \prime} \mathrm{N}$, $38^{\circ} 16^{\prime} 55^{\prime \prime}$ E, 01.09.2015.

50. Şebinkarahisar, E of Saryer village, woodland, $1598 \mathrm{~m}, 40^{\circ} 16^{\prime} 07^{\prime \prime} \mathrm{N}, 38^{\circ} 32^{\prime} 37^{\prime \prime} \mathrm{E}$, 01.09.2015.

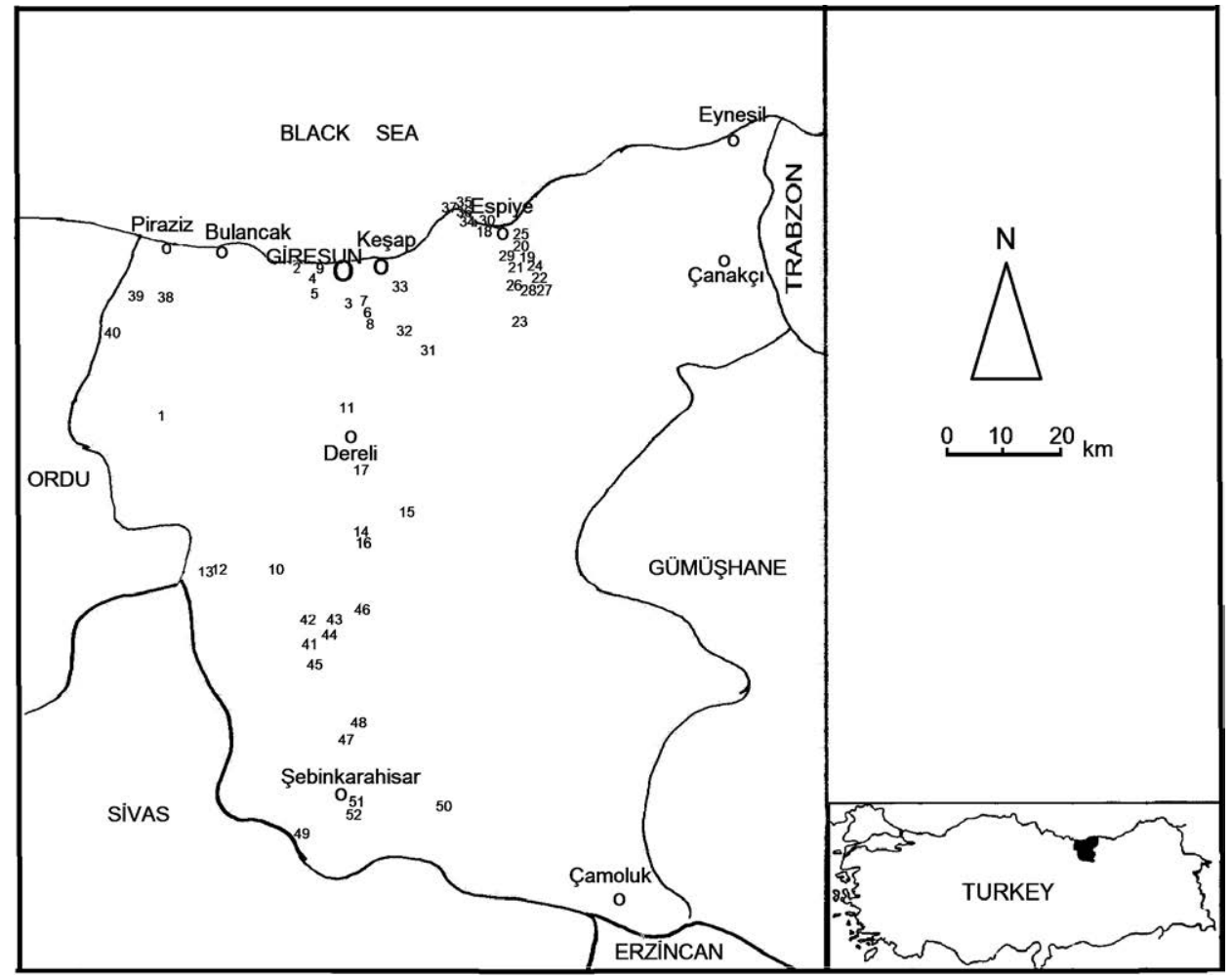

Fig. 1. Map of the collecting localities and numbers 
51. Şebinkarahisar, near the top of Şebinkarahisar castle, $1515 \mathrm{~m}, 40^{\circ} 17^{\prime} 05^{\prime \prime} \mathrm{N}, 38^{\circ} 25^{\prime} 42^{\prime \prime}$ E, 01.09.2015.

52. Şebinkarahisar, $\mathrm{N}$ slopes of Şebinkarahisar castle, $1515 \mathrm{~m}, 40^{\circ} 17^{\prime} 06^{\prime \prime} \mathrm{N}, 38^{\circ} 25^{\prime} 45^{\prime \prime} \mathrm{E}$, 01.09.2015.

\section{RESULTS}

An alphabetical list of the lichen species identified from the Giresun province is followed. First records for Turkey are marked by a hash symbol $(\#)$ and for Giresun by an asterisk $\left(^{*}\right)$.

*Acarospora badiofusca (Nyl.) Th. Fr. - Loc. 43 (GUB-6227): siliceous rock.

\#Acarospora molybdina (Wahlenb.) Trevisan - (Fig. 2) - Loc. 49 (GUB6228): calcareous rock. - Thallus light brown. Apothecia immersed, mostly single, rarely a few per areole, disc dark brownish, to $1.8 \mathrm{~mm}$ diam. Epihymenium yellowish brown, hypothecium yellowish, hymenium 60-150 $\mu \mathrm{m}$ tall, I+ blue. Ascospores simple, colourless, rounded, ellipsoid, cylindrical, 2.5-5 $\mu \mathrm{m}$ $\times 2.25-2.5 \mu \mathrm{m}$. Thallus $\mathrm{C}_{-}, \mathrm{K}-, \mathrm{KC}-$, PD-.

A detailed descriptions of the species is given in Thomson (1997). Acarospora molybdina differs by its longer lobes and mostly sessile apothecia, but is

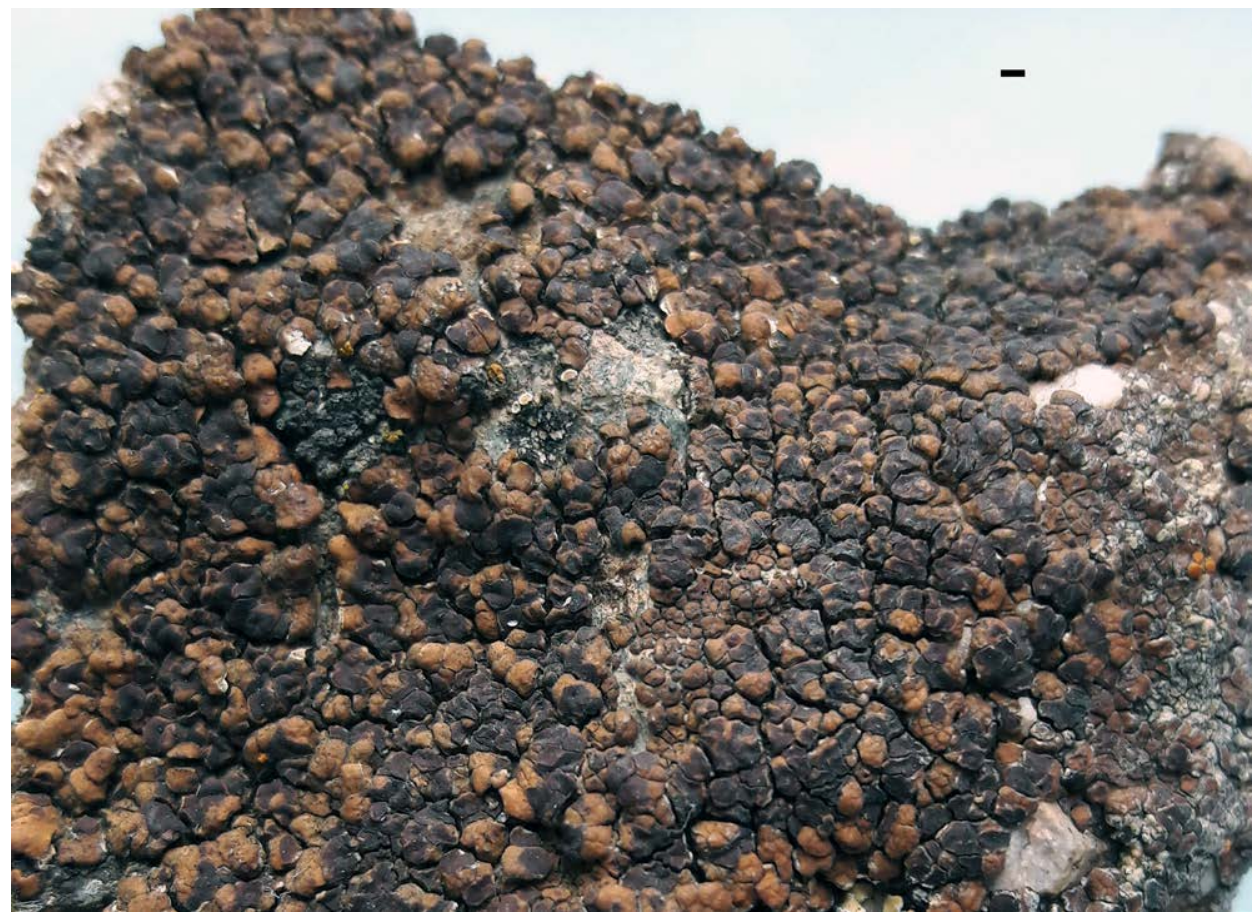

Fig. 2. Acarospora molybdina habit (scale bar $=1 \mathrm{~mm})$ 
more polymorphic than the samples of $A$. macrocyclos we examined (Knudsen et al. 2012). The Turkish material of A. molybdina differs from North American specimens by having bigger ascospores and a lower hymenium. In the North American collections the ascospores is $3-4 \times 1.5 \mu \mathrm{m}$, and the hymenium is 90-185 $\mu \mathrm{m}$ high (Thomson 1997). The Turkish collection differs ecologically in occurring on calcareous rock. A. molybdina is a circumpolar arctic species, growing on acid rocks, and particularly on boulders manured by birds along coasts (Thomson 1997). Known from Norway, Sweden and the United States (Knudsen et al. 2012, Thomson 1997). In Turkey, it was collected from sunny calcareous rocks at an elevation of $1037 \mathrm{~m}$ near the Kılıçkaya dam in Şebinkarahisar County. This is the first report of the species from Turkey.

*Acarospora veronensis A. Massal. - Loc. 44 (GUB-6229): siliceous rock; Loc. 49 (GUB-6230): calcareous rock.

Acrocordia macrospora A. Massal. - Loc. 37 (GUB-6231): siliceous rock.

Anisomeridium polypori (Ellis et Everh.) M. E. Barr. - Loc. 23 (GUB-6232): Malus sp.

Arthonia cinnabarina (DC.) Wallr. - Loc. 22 (GUB-6233): Tilia sp.

*Arthonia punctiformis Ach. - Loc. 14 (GUB-6234): Rhododendron sp.; Loc. 9 (GUB-6235): Corylus sp.

Arthonia radiata (Pers.) Ach. - Loc. 14 (GUB-6236): Rhododendron sp.; Loc. 9 (GUB-6237): Corylus sp.

${ }^{*}$ Arthopyrenia cinereopruinosa (Schaer.) A. Massal. - Loc. 5 (GUB-6238): Corylus sp.

*Arthopyrenia salicis A. Massal. - Loc. 21 (GUB-6239): Fagus sp.

Arthopyrenia laevata (Ach.) Arnold - Loc. 10 (GUB-6240) and Loc. 40 (GUB-6241): siliceous rock.

*Bacidina sulphurella (Samp.) M. Hauck et V. Wirth - Loc. 19 (GUB-6242): wooden telephone pole.

${ }^{*}$ Bagliettoa calciseda (DC.) Gueidan et Cl. Roux - Loc. 1 (GUB-6243) and Loc. 32 (GUB-6244): calcareous rock.

Bellemerea cinereorufescens (Ach.) Clauzade et Cl. Roux - Loc. 44 (GUB6245): siliceous rock.

Blennothallia crispa (Huds.) Otálora, P. M. Jørg. et Wedin - Loc. 7 (GUB6246): mortar.

Bryoria fuscescens (Gyeln.) Brodo et D. Hawksw. - Loc. 46 (GUB-6247): moss.

*Buellia aethalea (Ach.) Th. Fr. - Loc. 37 (GUB-6248) and Loc. 50 (GUB6249): siliceous rock.

${ }^{*}$ Calicium abietinum Pers. - Loc. 24 (GUB-6250): garden fence.

${ }^{*}$ Caloplaca ceracea J. R. Laundon - Loc. 35 (GUB-6251): siliceous rock. 
Caloplaca erythrocarpia (Pers.) Zwackh - Loc. 30 (GUB-6252) and Loc. 37 (GUB-6253): siliceous rock.

Caloplaca obscurella (J. Lahm) Th. Fr. - Loc. 45 (GUB-6254): Quercus sp.

${ }^{*}$ Caloplaca pellodella (Nyl.) Hasse - Loc. 35 (GUB-6255), Loc. 37 (GUB6256), Loc. 42 (GUB-6257), Loc. 43 (GUB-6258) and Loc. 44 (GUB-6259): siliceous rock.

${ }^{*}$ Caloplaca schoeferi Poelt - Loc. 12 (GUB-6260) and Loc. 41 (GUB-6261): moss.

${ }^{*}$ Caloplaca stillicidiorum (Vahl) Lynge - Loc. 52 (GUB-6262): moss.

*Carbonea vorticosa (Flörke) Hertel - Loc. 41 (6263): siliceous rock.

${ }^{*}$ Catillaria nigroclavata (Nyl.) Schuler - Loc. 4 (GUB-6264): Alnus sp.

Chaenotheca trichialis (Ach.) Th. Fr. - Loc. 14 (GUB-6265): Pinus sp.

Cladonia cyathomorpha Stirt. ex Walt. Watson - Loc. 20 (GUB-6266): soil.

Cladonia humilis (With.) J. R. Laundon - Loc. 45 (GUB-6267): soil.

Cladonia pocillum (Ach.) O. J. Rich. - Loc. 52 (GUB-6268): soil.

Cladonia rei Schaer. - Loc. 28 (GUB-6269): moss. soil.

Circinaria calcarea (L.) A. Nordin, S. Savić et Tibell - Loc. 12 (GUB-6270):

${ }^{*}$ Circinaria gibbosa (Ach.) A. Nordin, S. Savić et Tibell - Loc. 35 (GUB6271): siliceous rock.

${ }^{*}$ Clauzadea monticola (Ach.) Hafellner et Bellem. - Loc. 39 (GUB-6272): siliceous rock.

Collema flaccidum (Ach.) Ach. - Loc. 52 (GUB-6273): moss.

Dermatocarpon luridum (With.) J. R. Laundon - Loc. 13 (GUB-6274): siliceous rock.

${ }^{*}$ Endocarpon pusillum Hedw. - Loc. 15 (GUB-6275) and Loc. 51 (GUB6276): soil.

Enterographa zonata (Körb.) Källsten ex Torrente et Egea - Loc. 9 (GUB6277): siliceous rock.

*Flavoplaca nigromarina (Vondrák, P. Ríha, Arup et Søchting) Arup, Søchting. et Frödén - Loc. 30 (GUB-6278) and Loc. 37 (GUB-6279): siliceous rock.

*Fuscidea cyathoides (Ach.) V. Wirth et Vězda - Loc. 36 (GUB-6280): siliceous rock.

*Fuscopannaria mediterranea (Tav.) P. M. Jørg. - Loc. 31 (GUB-6281): Platanus sp.

*Immersaria athroocarpa (Ach.) Rambold et Pietschm. - Loc. 45 (GUB6282): calcareous rock.

*Immersaria cupreoatra (Nyl.) Calatayud et Rambold - Loc. 44 (GUB-6283) and Loc. 52 (GUB-6284): siliceous rock.

${ }^{*}$ Lathagrium auriforme (With.) Otálora, P. M. Jørg. et Wedin - Loc. 52 (GUB-6285): calcareous rock. 
Lecania naegelii (Hepp) Diederich et van den Boom - Loc. 6 (GUB-6286): Ficus sp.

Lecanora carpinea (L.) Vain. - Loc. 17 (GUB-6387): Prunus sp.

Lecanora chlarotera Nyl. - Loc. 50 (GUB-6288): Corylus sp.

Lecanora intumescens (Rebent.) Rabenh. - Loc. 16 (GUB-6289): Fagus sp.

Lecanora saligna (Schrad.) Zahlbr. - Loc. 24 (GUB-6290): garden fence.

Lecanora subcarnea (Lilj.) Ach. - Loc. 41 (GUB-6291): calcareous rock.

${ }^{*}$ Lecanora subrugosa Nyl. - Loc. 38 (GUB-6292): Corylus sp.

Lecidea lapicida (Ach.) Ach. - Loc. 36 (GUB-6293): siliceous rock.

Lecidea lapicida var. pantherina Ach. - Loc. 45 (GUB-6294): iron-rich rock.

${ }^{*}$ Lecidea promiscens Nyl. - Loc. 15 (GUB-6295): siliceous rock.

Lecidella achristotera (Nyl.) Hertel et Leuckert - Loc. 4 (GUB-6296): Corylus sp.

${ }^{*}$ Lecidella asema (Nyl.) Knoph et Hertel - Loc. 36 (GUB-6297) and Loc. 44 (GUB-6298): siliceous rock.

${ }^{*}$ Lecidella patavina (A. Massal.) Knoph et Leuckert - Loc. 28 (GUB-6299) and Loc. 51 (GUB-6300): siliceous rock.

Lecidella stigmatea (Ach.) Hertel et Leuckert - Loc. 3 (GUB-6301): mortar.

${ }^{*}$ Lepraria caesioalba (de Lesd.) J. R. Laundon - Loc. 15 (GUB-6302): moss.

${ }^{*}$ Lobaria amplissima (Scop.) Forssell - Loc. 31 (GUB-6303): Platanus sp.

${ }^{*}$ Lobothallia melanaspis (Ach.) Hafellner - Loc. 48 (GUB-6304): calcareous rock.

${ }^{*}$ Lobothallia praeradiosa (Nyl.) Hafellner - Loc. 46 (GUB-6305) and Loc. 51 (GUB-9306): siliceous rock; Loc. 48 (GUB-6307): calcareous rock.

${ }^{*}$ Micarea melaena (Nyl.) Hedl. - Loc. 29 (GUB-6308): garden fence.

Micarea prasina Fr. - Loc. 24 (GUB-6309): dead tree.

*Moelleropsis nebulosa (Hoffm.) Gyeln. - Loc. 34 (GUB-6310): soil.

${ }^{*}$ Mycobilimbia berengeriana (A. Massal.) Hafellner et V. Wirth - Loc. 12 (GUB-6311): soil.

${ }^{*}$ Mycocalicium subtile (Pers.) Szatala - Loc. 19 (GUB-6312): garden fence.

Myriolecis albescens (Hoffm.) Śliwa, Zhao Xin et Lumbsch - Loc. 24 (GUB6313): garden fence.

Myriolecis dispersa (Pers.) Śliwa, Zhao Xin et Lumbsch - Loc. 7 (GUB6314): mortar.

Myriolecis hagenii (Ach.) Śliwa, Zhao Xin et Lumbsch - Loc. 35 (GUB6315): siliceous rock.

${ }^{*}$ Myriolecis perpruinosa (Fröberg) Śliwa, Zhao Xin et Lumbsch - Loc. 44 (GUB-6316): siliceous rock.

${ }^{*}$ Myriolecis semipallida (H. Magn.) Śliwa, Zhao Xin et Lumbsch - Loc. 44 (GUB-6317) and Loc. 47 (GUB-6318): siliceous rock. 
*Naeotrocymbe punctiformis (Pers.) R. C. Harris - Loc. 9 (GUB-6319): Corylus sp.

${ }^{*}$ Opegrapha lithyrga Ach. - Loc. 5 (GUB-6320): siliceous rock.

* Opegrapha cf. niveoatra (Borrer) J. R. Laundon - Loc. 34 (GUB-6321): Corylus sp.

Opegrapha rufescens Pers. - Loc. 17 (GUB-6322): Prunus sp.

*Parvoplaca tiroliensis (Zahlbr.) Arup, Søchting et Frödén - Loc. 51 (GUB6323): plant debris.

Pertusaria corallina (L.) Arnold - Loc. 37 (GUB-6324): siliceous rock.

*Pertusaria cf. leioplaca DC. - Loc. 25 (GUB-6325): Laurocerasus sp.

Pertusaria leucosora Nyl. - Loc. 17 (6326): moss; Loc. 47 (GUB-6327): siliceous rock.

Physcia caesia (Hoffm.) Hampe ex Fürnr. - Loc. 52 (GUB-6328): moss.

*Physconia detersa (Nyl.) Poelt - Loc. 51 (GUB-6329): siliceous rock; Loc. 52 (GUB-6330): moss.

*Physconia grisea (Lam.) Poelt - Loc. 51 (GUB-6331): moss.

*Physconia muscigena var. petraea Poelt - Loc. 15 (GUB-6332): siliceous rock.

*Placidium lachneum (Ach.) de Lesd. - Loc. 15 (GUB-6333): moss.

*Placidium cf. rufescens (Ach.) A. Massal. - Loc. 42 (GUB-6334): soil.

*Placopyrenium fuscellum (Turner) Gueidan et Cl. Roux - Loc. 6 (GUB6335): mortar.

*Placopyrenium cf. iranicum Breuss - Loc. 42 (GUB-6336): moss.

*Placynthiella icmalea (Ach.) Coppins et P. James - Loc. 15 (GUB-6337): soil.

*Placynthiella uliginosa (Schrad.) Coppins et P. James - Loc. 15 (GUB6338): soil.

Placynthium nigrum (Huds.) Gray - Loc. 1 (GUB-6339): mortar; Loc. 37 (GUB-6340): siliceous rock.

Polysporina simplex (Taylor) Vězda - Loc. 15 (GUB-6341): siliceous rock.

*Porina ahlesiana (Körb.) Zahlbr. - Loc. 30 (GUB-6342): siliceous rock.

Porpidia macrocarpa (DC.) Hertel et A. J. Schwab - Loc. 11 (GUB-6343): siliceous rock.

Porpidia tuberculosa (Sm.) Hertel et Knoph - Loc. 45 (GUB-6344): iron-rich rock.

Protoblastenia rupestris (Scop.) J. Steiner - Loc. 39 (GUB-6345): siliceous rock. rock.

Protoparmelia badia (Hoffm.) Hafellner - Loc. 45 (GUB-6346): iron-rich

*Protoparmeliopsis garovaglii (Körb.) Arup, Zhao Xin et Lumbsch - Loc. 45 (GUB-6347): siliceous rock. 
*Pseudephebe pubescens (L.) M. Choisy - Loc. 46 (GUB-6348): siliceous rock.

${ }^{*}$ Psora globifera (Ach.) A. Massal. - Loc. 15 (GUB-6349): soil.

${ }^{*}$ Psorotichia schaereri (A. Massal.) Arnold - Loc. 1 (GUB-6350): siliceous rock. rock.

Pyrenodesmia variabilis (Pers.) A. Massal. - Loc. 49 (GUB-6351): calcareous

Pyrenula subelliptica (Tuck.) R. C. Harris - Loc. 22 (GUB-6352): Laurocerasus sp.

Rhizocarpon geographicum (L.) DC. - Loc. 37 (GUB-6353): siliceous rock.

${ }^{*}$ Rhizocarpon oederi (Weber) Körb. - Loc. 45 (GUB-6354): iron-rich rock.

${ }^{*}$ Rhizocarpon reductum Th. Fr. - Loc. 18 (GUB-6355): siliceous rock.

Rinodina atrocinerea (Hook.) Körb. - Loc. 43 (GUB-6356): siliceous rock.

${ }^{*}$ Rinodina bischoffii (Hepp) A. Massal. - Loc. 49 (GUB-6357): calcareous rock.

${ }^{*}$ Rinodina milvina (Wahlenb.) Th. Fr. - Loc. 42 (GUB-6358): siliceous rock.

${ }^{*}$ Rinodina oleae Bagl. - Loc. 29 (GUB-6359): Carpinus sp.

Rufoplaca arenaria (Pers.) Arup, Søchting et Frödén - Loc. 44 (GUB-6360): siliceous rock.

*Sarcogyne privigna (Ach.) A. Massal. - Loc. 18 (GUB-6361): siliceous rock.

Sarcogyne regularis Körb. - Loc. 4 (GUB-6362) and Loc. 44 (GUB-6363): siliceous rock.

Schaereria fuscocinerea (Nyl.) Clauzade et Cl. Roux - Loc. 43 (GUB-6364): siliceous rock.

Schismatomma decolorans (Sm.) Clauzade et Vězda - Loc. 5 (GUB-6365): siliceous rock.

Scoliciosporum umbrinum (Ach.) Arnold - Loc. 15 (GUB-6366): siliceous rock; Loc. 17 (GUB-6367): Prunus sp.

Staurothele areolata (Ach.) Lettau - Loc. 44 (GUB-6368): siliceous rock.

*Staurothele frustulenta Vain. - Loc. 49 (GUB-6369): calcareous rock and concrete.

*Staurothele rufa (A. Massal.) Zschacke - Loc. 45 (GUB-6370): siliceous rock.

Strigula glabra (A. Massal.) V. Wirth - Loc. 20 (GUB-6371): Corylus sp.

Thallinocarpon nigritellum (Lettau) P. M. Jørg. - Loc. 51 (GUB-6372): siliceous rock.

*Thelenella muscorum (Fr.) Vain. - Loc. 41 (GUB-6373): moss.

Trapelia coarctata (Sm.) M. Choisy - Loc. 4 (GUB-6374), Loc. 26 (GUB6375) and Loc. 27 (GUB-6376): siliceous rock.

${ }^{*}$ Trapeliopsis granulosa (Hoffm.) Lumbsch - Loc. 29 (GUB-6377): garden fence. 
*Umbilicaria polyphylla (L.) Baumg. - Loc. 50 (GUB-6378): siliceous rock. Umbilicaria subglabra (Nyl.) Harm. - Loc. 42 (GUB-6379): siliceous rock. Usnea hirta (L.) F. H. Wigg. - Loc. 5 (GUB-6380): siliceous rock. *Usnea cf. lapponica Vain. - Loc. 50 (GUB-6381): Quercus sp. Vahliella leucophaea (Vahl) P. M. Jørg. - Loc. 15 (GUB-6382): moss.

*Verrucaria latericola (Erichsen) Nav.-Ros. et Cl. Roux - Loc. 48 (GUB6384): calcareous rock.

Verrucaria muralis Ach. - Loc. 8 (GUB-6385): mortar.

Verrucaria nigrescens Pers. - Loc. 7 (GUB-6386): mortar.

*Verrucaria ochrostoma Borrer - Loc. 32 (GUB-6387): limestone; Loc. 42 (GUB-6388): siliceous rock.

*Verrucaria polysticta Borrer - Loc. 48 (GUB-6389) and Loc. 49 (GUB-6390): calcareous rock; Loc. 50 (GUB-6391): siliceous rock.

*Verrucaria viridula (Schrad.) Ach. - Loc. 49 (GUB-6392): calcareous rock. *Verrucula dolosa Hepp - Loc. 33 (GUB-6383): siliceous rock.

Altogether 136 lichen taxa were yielded (134 species and 2 varieties) from Giresun province. The 109 taxa of them represent a crustose growth form, 15 represent a foliose growth form, 11 represent a fruticose growth form, and 1 represents leprose growth form.

A 73 species (54.08\%) are new records for the Giresun province and Acarospora molybdina is new for the Turkish lichen biota.

Anisomeridium polypori, Caloplaca schoeferi, Lecanora perpruinosa, Moelleropsis nebulosa, Placopyrenium cf. iranicum, Placynthiella uliginosa and Rhizocarpon oederi were recorded for the second time in Turkey (John and Breuss 2004, Kocakaya et al. 2009, Şenkardeşler and Sukatar 2006, Vondrák et al. 2016, Yazıc1 1999, Yazıc1 and Aptroot 2008, Yazıc1 et al. 2015), while Aspicilia gibbosa, Bacidina sulphurella (Güvenç et al. 2006, Yazıcı et al. 2015; as Bacidina arnoldiana (Körb.) V. Wirth et Vězda), Flavoplaca nigromarina, Cladonia cyathomorpha, Lecidea berengeriana (Kınalığlu 2010b: as Mycobilimbia berengeriana (A. Massal.) Hafellner et V. Wirth), Opegrapha cf. niveoatra, Porina ahlesiana (Steiner 1899: as Verrucaria ahlesiana (Zwackh) Nik. Hoffm. et Hafellner), and Schismatomma decolorans, for the third time in Turkey (Kınalığlu, 2010b, d, Kınalıoğlu and Aptroot 2010, Güvenç et al. 2006, Özdemir Türk and Güner 1998, Yazıcı and Aslan 2003, Steiner 1899, 1916, Szatala 1927, Vondrák et al. 2009, Yazıc1 and Aptroot 2007, 2008, Yazic1 et al. 2010, 2011).

The most rich 4 genera in the study area are Lecanora (6 species), Myriolecis (6 species), Verrucaria (6 species) and Rinodina (5 species).

From a total of 136 lichen taxa, 78 were saxicolous, 33 epiphytic, 12 muscicolous and 11 terricolous. In addition, one taxon was both muscicolous and 
saxicolous (Physconia detersa) and one taxon corticolous and saxicolous (Scoliciosporum umbrinum).

The collecting site nr. 15 is characterised by the highest number of taxa (11 taxa), site nrs 37 and 44 with 9-9 taxa and site nr. 49 with 8 taxa.

According to the studies from Giresun province, 475 lichen taxa are recorded so far. After our contribution, the number of lichen taxa known in the province reaches now to 548. The knowledge of lichen biota of Giresun province may be considered as nearly complete as a result of this research. However, due to the high lichen diversity of the province, additional records might occur during future studies.

\section{REFERENCES}

Aslan, A. and Yazıc1, K. (2006): Contribution to the lichen flora of Giresun Province of Turkey. - Acta Bot. Hung. 48(3-4): 231-245. https://doi.org/10.1556/ABot.48.2006.3-4.1 Aslan, A., Aptroot, A. and Yazıc1, K. (2002): New lichens for Turkey. - Mycotaxon 84: 227-280.

Brodo, I. M., Sharnoff, S. D. and Sharnoff, S. (2001): Lichens of North America. - Yale University Press, New Haven and London, 795 pp.

Çobanoğlu, G. (2011): Additional and new lichen records for the province of Giresun. Marmara Üniversitesi Fen Bilimleri Dergisi 23(2): 83-88.

Dobson, F. S. (2011): Lichens: an illustrated guide to the British and Iris species. - Richmond Publishing, Slough, $496 \mathrm{pp}$.

Duman, C. and Yurdakulol, E. (2007): Lichen records from Sarıçiçek Mountain in Southern Giresun Province, Turkey. - Turk. J. Bot. 31: 357-365.

Halıc1, M. G. and Şenkardeşler, A. (2009): Giresun için yeni kayıt: Phaeosporobolus usneae. - T. LT. Bül. 7: 11-12.

Güvenç, Ş., Öztürk Ş. and Aydın S. (2006): Contributions to the lichen flora of Kastamonu and Sinop Provinces in Turkey. - Nova Hedwigia 83(1-2): 67-98. https://doi. org/10.1127/0029-5035/2006/0083-0067

John, V. (2007): Lichen Anatolici Exsiccati. - Arnoldia 26, Fasc. 8 (no. 176-200).

John, V. and Breuss, O. (2004): Flechten der östlichen Schwarzmeer-Region in der Türkei (BLAM Exkursion 1997). - Herzogia 17: 137-156.

Kınalıoğlu, K. (2005): Lichens of Giresun District, Giresun Province, Turkey. - Turk. J. Bot. 29: 417-423.

Kınalığlu, K. (2006): Lichens of Keşap District (Giresun, Turkey). - Acta Bot. Hung. 48(1-2): 65-76. https://doi.org/10.1556/ABot.48.2006.1-2.9

Kınalığlu, K. (2008): Three new records for the lichen biota of Turkey. - Mycotaxon 103: 123-126.

Kınalıŏlu, K. (2009): Additional lichen records from Giresun Province, Turkey. - Mycotaxon 109: 137-140. https://doi.org/10.5248/109.137

Kınalıŏlu, K. (2010a): Cladonia, Lecanographa, Ochrolechia, and Placidium species new to Turkey. - Myxotaxon 113: 203-208. https://doi.org/10.5248/113.203

Kınalıŏlu, K. (2010b): New and interesting records of lichens from Turkey. - Myxotaxon 114: $85-90$. https://doi.org/10.5248/114.85 
Kınalığlu, K. (2010c): Five new records for the lichen biota of Turkey. - Myxotaxon 112: 371-375. https://doi.org/10.5248/112.371

Kınalığlu, K. (2010d): Epiphytic and saxicolous lichens of district centre of Arakli and in vicinity (Trabzon, Turkey). - Pakistan J. Biol. Sci. 13(12): 588-595. https://doi. org/10.3923/pjbs.2010.588.595

Kınalığlu, K. and Aptroot, A. (2010): Catillaria, Cladonia, Strigula, and Cresporhaphis species new to Turkey and Asia. - Myxotaxon 114: 329-332. https://doi.org/105248/114.329

Kınalığlu, K. and Aptroot, A. (2011): Carbonea, Gregorella, Porpidia, Protomicarea, Rinodina, Solenopsora, and Thelenella lichen species new to Turkey. - Myxotaxon 115: 125-129. https://doi.org/10.5248/115.125

Kınalığlu, K. and Engin, A. (2004): Bülbülan (Artvin), Ayder, Anzer (Rize), Kalecik (Trabzon) ve Kümbet (Giresun) Yaylalarının Likenleri. - Ot Sistematik Botanik Dergisi (The Herb J. Syst. Bot.) 11(2): 167-190.

Kınalığlu, K. and Uzun, G. (2016): Şebinkarahisar (GİRESUN) İlçesinden Liken ve Likenikol Mantar Kayıtları. (Lichenized and lichenicolous fungi records from Şebinkarahisar District (GİRESUN)). - AKÜ FEMÜBİD 16 (011003): 16-25. https:// doi.org/10.5578/fmbd.25264

Kocakaya, M., Halıc1, M. G. and Aksoy, A. (2009): Lichens and lichenicolous fungi of Kizıldağ (Derebucak, Konya). - Turk. J. Bot. 33: 105-112. https://doi.org/10.3906/bot0810-2

Küçük, M. (1990): Giresun Adası'nın Floristik Yapısı. - Ormancılık Araştırma Enstitüsü Yaymları 36(2): 58.

Knudsen, K., Flakus, A. and Kukwa, M. (2012): A contribution to the study of Acarosporaceae in South America. - Lichenologist 44(2): 253-262. https://doi.org/10.1017/ s0024282911000703

Özdemir Türk, A. and Güner, H. (1998): Lichens of the Thrace region of Turkey. - Turk. J. Bot. 22: 397-407.

Şenkardeşler, A. and Sukatar, A. (2006): Lichens of Denizli. - JFS 29: 52-66.

Sezer, O. (2016): Türkiye liken biyotasına katkılar. - AÇU Orman Fak. Derg. 17(1): 70-81.

Smith, C. W., Aptroot, A., Coppins, B. J., Fletcher, A., Gilbert, O. L., James, P. W. and Wolseley, P. A. (2009): The lichens of Great Britain and Ireland. - British Lichen Society, London, $1046 \mathrm{pp}$.

Steiner, J. (1899): Lichenes. In: Fritsch, K.: Beitrag zur Flora von Constantinopel, Bearbeitung der von J. Nemetz in den Jahren 1894-1897 in den Umgebung von Constantinopel gesammelten Pflanzen. I. Kryptogamen. - Denkschr. Kaiserl. Akad. Wiss., Wien. Math.-Naturwiss. Kl. 68: 219-249.

Steiner, J. (1909): Lichenes. In: Handel-Mazzetti, D. H. F. V.: Ergebnisse einer botanischen Reise in das Pontische Randgebirge im Sandschak Trapezunt, etc. - Ann. Naturhist. Hofmuseum Wien 23: 107-123.

Steiner, J. (1916): Aufzählung der von J. Bornmüller im Oriente gesammelten Flechten. Ann. Naturhist. Hofmuseum Wien 30: 24-39.

Szatala, Ö. (1927): Lichenes in Asia minore ab directore Dre Stefano Györffy de Szigeth (Budapest) et Dre Andrasovszky collecti. - Folia Cryptog. 1: 272-278.

Thomson, J. W. (1997): American arctic lichens. 2. The microlichens. - The University of Wisconsin Press, Madison, 675 pp.

Vondrák, J., К̌íha, P., Arup, U. and Søchting, U. (2009): The taxonomy of the Caloplaca citrina group (Teloschistaceae) in the Black Sea region; with contributions to the cryptic 
species concept in lichenology. - Lichenologist 41(6): 571-604. https://doi.org/10.1017/ S0024282909008317

Vondrák, J., Halıcı, M. G., Güllü, M. and Demireli, R. (2016): Taxonomy of the genus Athallia and its diversity in Turkey. - Turk. J. Bot. 40: 319-328. https://doi.org/10.3906/bot1502-12

Wasser, S. P. and Nevo, E. (2005): Lichen-forming, lichenicolous, and allied fungi of Israel. - A. R. G. Gantner Verlag K. G., Ruggell, 384 pp.

Wirth, V. (1995): Die Flechten Baden-Württembergs. Teil 1-2. - Ulmer, Stuttgart, 1006 pp.

Wirth, V., Hauck, M. and Schultz, M. (2013): Die Flechten Deutschlands. - Ulmer, Stuttgart, $1244 \mathrm{pp}$.

Yazıc1, K. (1999): Lichen flora of Trabzon. - Turk. J. Bot. 23: 97-112.

Yazic1, K. and Aptroot, A. (2007): Five lichen species new to Turkey. - Mycotaxon 100: 21-26.

Yazıc1, K. and Aptroot, A. (2008): Corticolous lichens of the city of Giresun with descriptions of four species new to Turkey. - Myxotaxon 105: 95-104.

Yazıc1, K. and Aslan, A. (2003): Lichens from the regions of Gümüşhane, Erzincan and Bayburt (Turkey). - Cryptogamie, Mycol. 24(2): 287-300.

Yazıcı, K. and Aslan, A. (2005): Six new lichen records from Turkey. - Myxotaxon 93: 359-363.

Yazıc1, K. and Aslan, A. (2006): Four new lichens from Turkey. - Myxotaxon 95: 315-318.

Yazıcı, K., Aptroot, A., Aslan, A., Etayo, J., Spier, L. and Karagöz, Y. (2010): Lichenized and lichenicolous fungi from nine different areas in Turkey. - Myxotaxon 111: 113-116. https://doi.org/10.5248/111.113

Yazıcı, K., Aptroot, A., Aslan, A., Vitikainen, O. and Piercey-Normore, M. D. (2011): Lichen biota of Ardahan province (Turkey). - Myxotaxon Link page: 116: 480. https://doi. org/10.5248/116.479; Regional Checklists online (http://www.mycotaxon.com/resources/checklists/Yazici-v116-checklist.pdf)

Yazıc1, K., Aptroot, A., Aslan, A., Sipman, H. and Piercey-Normore, M. D. (2015): The Lichen biota of Burdur Province (Turkey). - Myxotaxon Link page: 130: 926. https://doi. org/10.5248/130.925; Regional Checklists online (http://www.mycotaxon.com/resources/checklists/Yazici-v130-3-checklist.pdf) 